\title{
Analysis of transmit power setting technique for cognitive radio networks
}

\author{
Kenta Umebayashi ${ }^{*}$, Janne Lehtomäki ${ }^{2}$ and Isameldin M. Suliman ${ }^{3}$
}

\begin{abstract}
We investigate a method to set the maximum allowable transmit power for a secondary base station in dynamic spectrum sharing among secondary users and primary users. In conventional methods, location information is assumed. Thus, the maximum allowable transmit power can be set by considering the shadowing between the secondary base station and the primary user receivers to satisfy a constraint. Specifically, the probability that the interference from secondary base station exceeds the acceptable level must be less than the constraint target probability. We assume that the location information is not available at the secondary network. Instead, the secondary base station uses the received signal strength from the primary user transmitter for distance estimation. In this case, we have to consider shadowing not only between the secondary base station and the primary user receivers, but also between the primary user transmitter and the secondary receiver(s). We also need to account for the uncertainty of the distance. In order to satisfy the constraint target probability, we proposed a two-step approach to setting the maximum allowable transmit power where a transmission decision margin and a transmit power margin are utilized. To reduce these margins, we also propose cooperative maximum allowable transmit power setting method utilizing also received signal strength values from several secondary users. Simulation results confirm the validity of the analysis and show the effectiveness of the proposed cooperative maximum allowable transmit power setting method, i.e., the capacity based on cooperative maximum allowable transmit power setting method is significantly better than that of non-cooperative maximum allowable transmit power setting method. In addition, we show a proper size of radius of additional separation area to protect primary users by the numerical results.
\end{abstract}

Keywords: Cognitive radio, Cooperative spectrum measurement, Transmit power setting, Spectrum sharing

\section{Introduction}

Spectrum scarcity is one of the most pressing problems in the field of wireless communications today. Since most of the available spectrum has already been exclusively assigned to licensed wireless systems, there is not much left for emerging wireless services. On the other hand, it has been reported that large portions of the assigned spectrum in both the time and space domains are underutilized by licensed users, also called primary users (PUs). This unused spectrum leads to temporal and geographic white spaces [1].

To overcome the spectrum scarcity problem, two promising techniques are used: opportunistic spectrum access (OSA) and spectrum sharing (SS), with cognitive

\footnotetext{
*Correspondence: ume_k@cc.tuat.ac.jp

1 Department of Electrical and Electronic Engineering, Tokyo University of

Agriculture and Technology, Tokyo, Japan

Full list of author information is available at the end of the article
}

radio techniques performed by secondary users (SUs) [2-6]. In the OSA approach, SUs are allowed to transmit over a spectrum originally assigned to PUs only if the PUs are not utilizing the spectrum [7-10].

To find such spectrum holes or white spaces, SUs generally employ spectrum sensing $[11,12]$. In comparison, in the SS approach, SUs can use the spectrum even if the $\mathrm{PU}$ is active with a constraint on interference at the PU. This constraint is usually defined by the interference level at the PU receiver and/or the outage probability, which is defined as the probability that the interference level is beyond a given threshold. In general, the SS approach has the potential to achieve greater spectrum utilization since concurrent transmissions by PUs and SUs are allowed. In comparison, only orthogonal transmissions are permitted by OSA [5].

A comparison between transmit power setting based on soft decisions (corresponding to SS) and transmit

\section{Springer Open}

(C) 2016 Umebayashi et al. Open Access This article is distributed under the terms of the Creative Commons Attribution 4.0 International License (http://creativecommons.org/licenses/by/4.0/), which permits unrestricted use, distribution, and reproduction in any medium, provided you give appropriate credit to the original author(s) and the source, provide a link to the Creative Commons license, and indicate if changes were made. 
power setting based on hard decisions (corresponding to OSA) was shown [13]. The comparison demonstrated that transmit power adaptation based on a soft decision can maximize the capacity of the SU.

In some research related to resource allocation with SS, it has been shown that transmit power control can improve the efficiency of spectrum utilization and protect PUs [14-24]. In fact, proper transmit power control can protect PUs without spectrum sensing and increase opportunities of SS by SUs [20].

In many of the works considering resource allocation, perfect instantaneous channel state information (CSI) or channel gain (in the link from the SU transmitter to the PU receiver) was assumed to be available [14-18]. There are several methods that could enable SUs to obtain the CSI. For example, the CSI could be periodically measured with a band manager or by using feedback from the PU receiver to the SUs [25]. However, support from the PU receiver or a third entity is necessary and such support is not always available.

In $[6,26]$, techniques to observe the behavior or reaction of the PUs have been proposed. For example, in [6], a SU sends a probing signal to intentionally interfere with the PU. The observed PU reactions, such as rate and/or power adaptation as well as automatic repeat request feedback, are used by the SU to learn the environment and set the transmission protocol for adapting to the environment.

Instead of the instantaneous CSI, several works employ location information (i.e., the distance between the SU transmitter and the PU receiver corresponding to an observation equipment for the distance) for transmit power control. Most of the works, it assumed that location information is available [19-23]. In [27-29], they assume that location information is available through a database and global positioning system. Given the location information, the path loss (denoted by $L$ ) can be determined and the SU transmitter can set a maximum allowable transmit power (MATP) that gives sufficient PU protection using an appropriate margin as a countermeasure against uncertainty such as shadowing [27-29]. For setting margins appropriately, knowledge of statistics of uncertainties is required.

In $[24,30]$, transmit power control based on a soft decision was investigated. In this research, the statistics of the soft decision were assumed to be known by the SUs. Specifically, estimation error in terms of the distance is modeled by Gaussian random value in [24]. However, the statistics may depend on the distance between the SU transmitter and the PU receiver. Availability of the statistical information is equivalent to the distance between the PU and the SU being available at the SU side.

In [7, 31], $L$ is estimated based on measured information such as received signal strength (RSS) of PU signal and signal-to-noise power ratio (SNR). However, an effect of shadowing in the estimation was not considered sufficiently even thought it can significantly affect the observed RSS values.

Accurate distance estimation between radios has been investigated in ranging and localization. In [32,33], localization techniques for a cognitive radio network were investigated. Important issues in distance estimation are as follows: noise, multi-path fading, shadowing, and uncertainty of location estimation. Time domain averaging can suppress the effects of noise and multi-path fading $[34,35]$; therefore, these effects are not considered in this paper.

Motivated by the aforementioned research, in this paper, we investigate a method to set the MATP in the context of the SS for a secondary base station (SB) based on estimated distance between the SB and the PU transmitter. The SB will estimate the distance based on the RSS which is randomly fluctuating due to shadowing. Therefore, we have to consider not only shadowing in the link from the SB to the PU as in [27-29] but also shadowing in the link from the PU transmitter, to the secondary receivers, to achieve an appropriate margin. In an assumed scenario of this paper, the PU transmitter corresponds to the PU base station (PB). In addition, there is a new issue caused by the unavailability of distance information. Specifically, appropriate margin for the shadowing effects can be obtained based on the knowledge of statistics of uncertainties, such as distance estimation; however, the statistic depends on the actual distance even though the actual distance is unavailable. We will show that the proposed MATP setting can overcome the contradiction.

A constraint for protecting $\mathrm{PU}$ is set for the constraint target probability (CTP) where a probability that the interference level caused by SB exceeds the allowable interference level should be less than CTP. Our main contributions to the literature are summarized as follows:

1. For the issue of the unavailability of distance information, we propose a two-step approach to setting the MATP. In fact, the two steps consist not only of the transmit power setting but also of the transmission decision and the both steps are performed based on distance estimation. In the transmit power setting and transmission decision, transmit power margin, $T_{\mathrm{m}}$ and transmission decision margin, $d_{\mathrm{m}}$, are used, respectively.

2. We provide a theoretical analysis of statistics in distance estimation and interference levels at the PU under a log-normal shadowing environment. We consider shadowing effects not only between the SB and the PU receivers but also between the $\mathrm{PB}$ and the secondary receivers (SUs and $\mathrm{SB}$ ). We then derive the appropriate margins, $d_{\mathrm{m}}$ and $T_{\mathrm{m}}$, based on this analysis. 
3. Numerical results will show that the SU network (SN) throughput depends on the additional separation radius which is used for protection of PUs. The SN consists of SB and SU terminals. For example, without the additional separation radius, the transmit power margins are significantly increased. This fact implies an existence of optimal additional separation radius, and this will also be confirmed by the numerical results.

4. We propose the MATP setting method based on cooperative spectrum measurement to achieve smaller margins. The simulation results verify that the MATP setting method based on cooperative spectrum measurement improves the spectrum utilization compared to the MATP setting using spectrum measurement results only from the $\mathrm{SB}$ (MATP setting method based on individual spectrum measurement).

The rest of this paper is organized as follows. In Section 2, the system model and assumptions are shown. In Section 3, the proposed MATP setting methods are described. Section 4 shows the analyses of the proposed MATP settings methods with a view to set the optimal margins. Numerical results are given in Section 5. The paper concludes with Section 6 .

The nomenclature list for most of the symbols and abbreviations used in this paper is shown in Table 1.

\section{System model and assumptions}

In this section, we introduce the framework used throughout the paper. The network model shown in Fig. 1 is discussed in Section 2.1, and the MATP setting assuming provided PU location information is explained in Section 2.2.

\section{$2.1 \quad$ Network model}

As shown in Fig. 1, there are two networks: the PN and the SN. The PN consists of one central control station, such as a base station or broadcasting station, denoted by $\mathrm{PB}$ and with PUs corresponding to terminals. The $\mathrm{PN}$ is licensed to operate over a frequency band with bandwidth $B$. The coverage of the PN is given by a circle with a radius $r_{c, p}$, with the PB located at the center [36]. A PU receiver located at the edge of the coverage area corresponds to the worst-case scenario [7]. The distance $d$ is defined as $d=0$ at the location of the $\mathrm{PB}$, and the $\mathrm{PU}$ receiver lies at $d=r_{c, p}$. We define the extended PN coverage $d_{g}$ to consist of the actual coverage and an additional separation radius $\Delta d_{g}$ such that $d_{g}=r_{c, p}+\Delta d_{g}$. The main role of the additional separation radius $\Delta d_{g}$ is to protect PUs $[8,37]$. Thus, it is preferable that $\mathrm{SB}$ may not operate in the area where $d_{\mathrm{SU}_{0}}<d_{g}$.
Table 1 Nomenclature

\begin{tabular}{ll}
\hline General & \\
MATP & Maximum allowable transmit power \\
CTP & Constraint target probability \\
RSS & Received signal strength \\
PB, PN, SB, SN & Primary base station, primary network, secondary \\
$B$ & base station, secondary network \\
$P_{C}$ & Bandwidth for PN operation \\
$\dot{P}_{C}$ & Constraint probability in (4) \\
$(Z)$ & Constraint target probability (CTP) \\
& Type of MATP such as MATP-Z where $Z$ is $P$, \\
$\mu$ and $v$ & C, or I \\
$C_{\text {down }}$ & Mean and variance \\
$\bar{C}_{\text {down }}$ & Downlink capacity for SN
\end{tabular}

Location and distance

\begin{tabular}{|c|c|}
\hline$r_{C, p}$ & Radius of coverage of the PN \\
\hline$r_{C, S}$ & Radius of coverage of the SN \\
\hline$d$ & Distance from PB \\
\hline$\Delta d_{g}$ & Additional separation radius \\
\hline$d_{g}$ & $=r_{c, p}+\Delta d_{g}$ : radius of extended PN coverage \\
\hline$d_{\mathrm{PU}-S U_{n}}$ & Distance between the $\mathrm{PB}$ and the $S U_{n}$ \\
\hline$d \mathrm{SU}_{0}$ & Distance between the PB and the SB \\
\hline$\hat{d}_{\mathrm{SU}_{0}}^{(\mathrm{z})}$ & Estimated $d_{s \cup_{0}}$ \\
\hline$d_{m}\left(\operatorname{or} d_{m}^{(Z)}\right)$ & Transmit decision margin \\
\hline
\end{tabular}

Transmission, propagation, and reception

\begin{tabular}{|c|c|}
\hline$T_{\mathrm{PU}}$ & Transmit power of the $P B$ in $d B$ \\
\hline$T_{\mathrm{SU}_{0}}$ & Transmit power of the SB in $\mathrm{dB}$ \\
\hline$T_{S U_{0}, \max }^{(Z)}$ & MATP \\
\hline$T_{m}\left(\operatorname{or} T_{m}^{(Z)}\right)$ & Transmit power margin \\
\hline $\mathrm{D}_{T}$ & Binary transmit decision variable (0 or 1$)$ \\
\hline$L(d)$ & Path loss \\
\hline $\mathrm{XPU}_{0} \rightarrow \mathrm{SU}_{n}, \sigma_{X}$ & $\begin{array}{l}\text { Attenuation due to shadowing in the link } \\
\text { between } \mathrm{PU}_{0} \text { and } \mathrm{SU}_{n}\end{array}$ \\
\hline$\sigma_{x}^{2}$ & Variance of log-normal shadowing model \\
\hline$R \mathrm{PU}_{0} \rightarrow \mathrm{SU}_{n}$ & RSS level from the PB transmission at $\mathrm{SU}_{n}$ \\
\hline $\bar{R}_{\mathrm{PU}_{0} \rightarrow \mathrm{SU}_{n}}$ & RSS level without the shadowing effect \\
\hline$\hat{R}^{(Z)}$ & Estimated $\bar{R}_{\mathrm{PU}_{0} \rightarrow \mathrm{SU}_{0}}$ \\
\hline IPU & $\begin{array}{l}\text { Interference level at the } \mathrm{PU} \text { receiver at the edge } \\
\text { of PN coverage }\end{array}$ \\
\hline $\bar{l}_{\mathrm{PU}}$ & Interference level without the shadowing effect \\
\hline Ith & Allowable interference level at the PU \\
\hline \multicolumn{2}{|l|}{$\mathrm{U}$ and SU } \\
\hline $\mathrm{PU}_{0}$ & PB \\
\hline$S U_{0}$ & SB \\
\hline $\mathrm{SU}_{n}\left(n=1, \cdots, \mathrm{N}_{s}\right)$ & $n$th SU terminal \\
\hline$N_{S}$ & Number of SU terminals \\
\hline
\end{tabular}




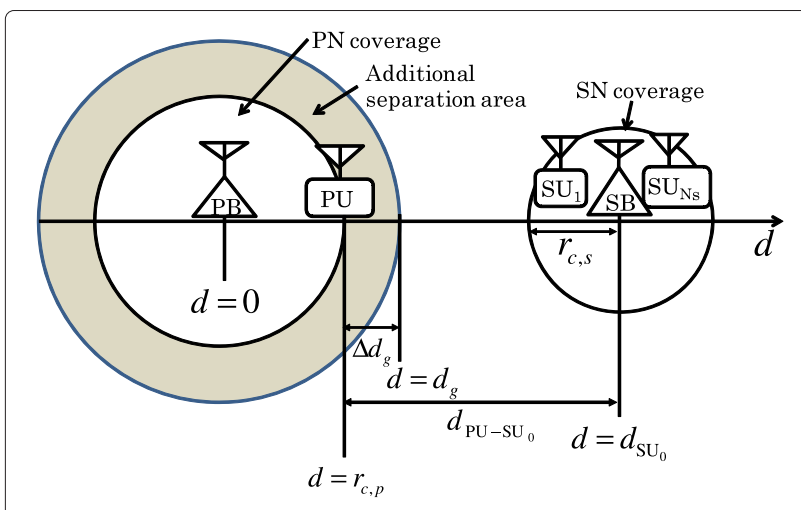

Fig. 1 The network model. The PU is located at the edge of the PB coverage

The SN consists of one base station, $\mathrm{SB}$, and $N_{s}$ SUs (terminals). The SB location is defined as $d=d_{\mathrm{SU}_{0}}$, and the radius of the SN coverage is denoted by $r_{c, s}$. The SUs are assumed to be uniformly distributed within the SN coverage.

In RSS measurement, the measured spectrum is dedicated to the $\mathrm{PB}$, such as broadcasting channel or downlink channel of the frequency division duplex. In addition, the averaging process in the measurement is assumed to suppress the effects of both multipath fading and additive white Gaussian noise. In both the broadcasting and downlink control channel, there may be continuous traffic and sufficiently long measurement time is assumed to provide accurate RSS level at the SUs.

The RSS level $R_{\mathrm{PU}_{0} \rightarrow \mathrm{SU}_{n}}$ from the PB transmission at the $n$th $\mathrm{SU}, \mathrm{SU}_{n}$, is given by

$$
\begin{aligned}
R_{\mathrm{PU}_{0} \rightarrow \mathrm{SU}_{n}} & =T_{\mathrm{PU}_{0}}-L\left(d_{\mathrm{SU}_{n}}\right)+X_{\mathrm{PU}_{0} \rightarrow \mathrm{SU}_{n}, \sigma_{x}} \\
& =\bar{R}_{\mathrm{PU}_{0} \rightarrow \mathrm{SU}_{n}}+X_{\mathrm{PU}_{0} \rightarrow \mathrm{SU}_{n}, \sigma_{x}},
\end{aligned}
$$

where $T_{\mathrm{PU}_{0}}$ is the transmit power of the $\mathrm{PB}$ in $\mathrm{dB}, L\left(d_{\mathrm{SU}_{n}}\right)$ is path loss in $\mathrm{dB}, d_{\mathrm{SU}_{n}}$ is the distance between the $\mathrm{PB}$ and the $\mathrm{SU}_{n}, X_{\mathrm{PU}_{0} \rightarrow \mathrm{SU}_{n}, \sigma_{x}}$ reflects the attenuation due to shadowing, and $\bar{R}_{\mathrm{PU}_{0} \rightarrow \mathrm{SU}_{n}}$ indicates the RSS without the shadowing effect. Note that the index $n=0$ is used for base stations. Without loss of generality, we assume that antenna gains both of the PB and SUs are 0 decibels relative to isotropic $(\mathrm{dBi})$. We employ the log-normal shadowing model [38] and assume that $X_{\mathrm{PU}_{0} \rightarrow \mathrm{SU}_{n}, \sigma_{x}}$ are independent and identically distributed (i.i.d) normal random variables with zero mean and variance $\sigma_{x}^{2}$. We assume a standard path loss model with a path loss exponent so that $L(d)$ is given by

$$
L(d)=10 \log _{10}\left(\frac{4 \pi d_{0}}{\lambda}\right)^{2}+10 \eta \log _{10}\left(\frac{d}{d_{0}}\right),
$$

where $d_{0}$ denotes a reference distance, $\lambda$ is the wavelength of the carrier frequency, and $\eta$ denotes the path loss exponent. Without loss of generality, we use $d_{0}=1 \mathrm{~m}$ throughout this paper. The coverage radii of the PN and the SN, $r_{c, p}$ and $r_{c, s}$, are set based on the minimum required received signal levels $\gamma_{\mathrm{PN}}$ and $\gamma_{\mathrm{SN}}$ at the PN and $\mathrm{SN}$, respectively.

A interference level $I_{\mathrm{PU}}$ at the PU receiver caused by the $\mathrm{SB}$ transmission is given by

$$
\begin{aligned}
I_{\mathrm{PU}} & =T_{\mathrm{SU}_{0}}-L\left(d_{\mathrm{PU}-\mathrm{SU}_{0}}\right)+X_{\mathrm{SU}_{0} \rightarrow \mathrm{PU}, \sigma_{x}} \\
& =\bar{I}_{\mathrm{PU}}+X_{\mathrm{SU}_{0} \rightarrow \mathrm{PU}, \sigma_{x}},
\end{aligned}
$$

where $T_{\mathrm{SU}_{0}}$ is the actual total transmit power of the $\mathrm{SB}$, $d_{\mathrm{PU}-\mathrm{SU}_{0}}$ is the distance between the $\mathrm{PU}$ receiver and the $\mathrm{SB}$, and $X_{\mathrm{SU}_{0} \rightarrow \mathrm{PU}, \sigma_{x}}$ is the log-normal shadowing effect in the link. The constraint probability that the interference level caused by SB exceeds the allowable interference level is given by

$$
\mathrm{P}_{\mathrm{C}}=\mathrm{P}_{\mathrm{r}}\left(I_{\mathrm{PU}}>I_{\mathrm{th}}\right)
$$

where $I_{\text {th }}$ indicate the allowable interference level at the PU. We set a constraint $\mathrm{P}_{\mathrm{r}}\left(I_{\mathrm{PU}}>I_{\text {th }}\right) \leq \dot{\mathrm{P}}_{\mathrm{C}}$, where $\dot{\mathrm{P}}_{\mathrm{C}}$ denotes the CTP. This constraint is used throughout this paper.

\subsection{Maximum allowable transmit power setting with provided location information}

To satisfy the CTP, the SB has to set an appropriate $T_{\mathrm{SU}_{0}}$.

In the conventional MATP setting approach, it is assumed that location information $\left(d_{\mathrm{SU}_{0}}\right)$ is known [27-29]. We denote this approach by MATP-P, where P stands for "provided location information," i.e., the SB knows the perfect location information. In the MATP-P, $d_{\mathrm{PU}-\mathrm{SU}_{0}}$, is also available at the SB since the SB knows $r_{c, p}[28,29]$. Given this information, MATP satisfying the constraint can be set as

$$
T_{\mathrm{SU}_{0}, \max }^{(P)}=I_{\mathrm{th}}+L\left(d_{\mathrm{PU}-\mathrm{SU}_{0}}\right)-\sigma_{x} Q^{-1}\left(\dot{\mathrm{P}}_{\mathrm{C}}\right),
$$

where the $P$ in $T_{\mathrm{SU}_{0}, \max }^{(P)}$ indicates the MATP-P, $Q^{-1}(x)$ is the inverse Q-function [39], and it is assumed that $\sigma_{x}$ is known at the SB. The term $\sigma_{x} Q^{-1}\left(\dot{\mathrm{P}}_{\mathrm{C}}\right)$ in (5) corresponds to a margin against the shadowing effect in a link from the SB to the PU. The MATP-P will be used as a reference method in order to see the loss due to distance estimation in the proposed approaches.

\section{Proposed MATP setting}

To satisfy the CTP with unknown distance to the PB, we propose a two-step approach with distance estimation for setting the MATP. A flowchart of this procedure is shown in Fig. 2.

The actual problem caused by the unavailability of distance information is shown as follows. In general, the 


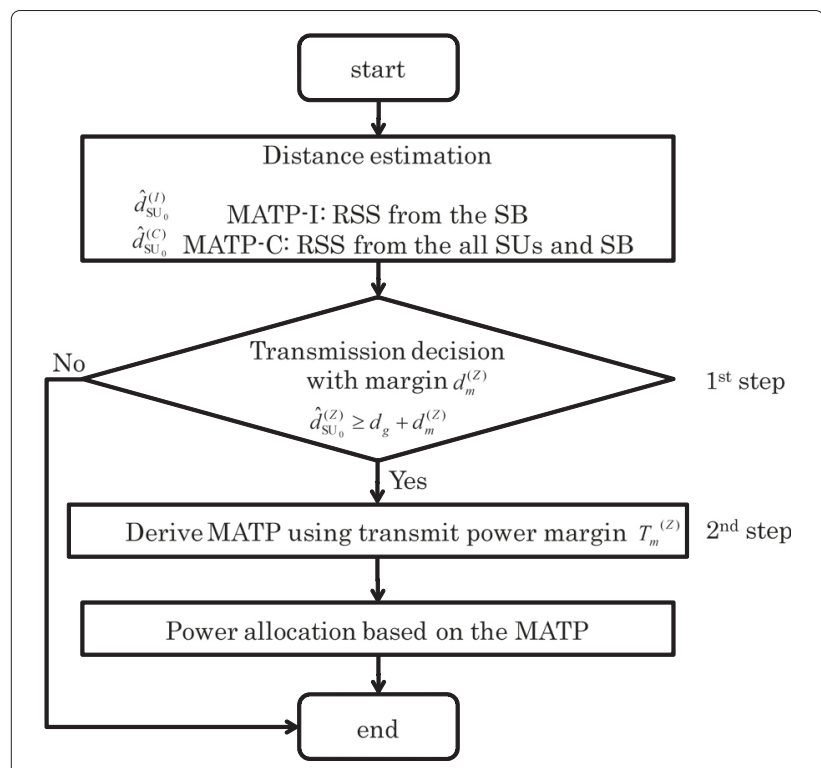

Fig. 2 A flowchart of the proposed MATP setting procedure. Superscript $(Z)$ indicates the type of MATP, i.e., $(P)$ for MATP-P $(C)$ for MATP-C, and (I) for MATP-I

transmit power margin $T_{\mathrm{m}}$ is set based on the worst-case scenario, i.e., the SB locates at the edge of extended PN coverage, $d_{\mathrm{SU}_{0}}=d_{g}$. In this case, the $T_{\mathrm{m}}$ can satisfy the CPT only when the $\mathrm{SB}$ is in the region where $d_{\mathrm{SU}_{0}} \geq d_{g}$. However, the SB may operate in the region where $d_{\mathrm{SU}_{0}}<$ $d_{g}$ since estimated distance is used. In this case, the CTP can not be satisfied. For overcoming this problem, the transmission decision with the decision margin is used to protect the PUs.

The procedure of the setting the MATP is as follows. The SN first calculates an estimate of the distance. The distance estimation is based on either the RSS value $R_{\mathrm{PU}_{0} \rightarrow \mathrm{SU}_{0}}$ collected by the $\mathrm{SB}$ alone (referred to as MATP-I, where I stands for "individual measurement") or the RSS values $R_{\mathrm{PU}_{0} \rightarrow \mathrm{SU}_{0}}$ and $R_{\mathrm{PU}_{0} \rightarrow \mathrm{SU}_{n}}$ collected by the $\mathrm{SB}$ and $N_{S}$ SUs $\left(n=1,2, \cdots, N_{S}\right)$ (referred to as MATP-C, where $C$ stands for "cooperative measurement").

The SB first decides if transmission is allowed by estimating whether the SB resides within extended PN coverage, $d_{g}$. The SB arrives at a decision by comparing the estimated distance to the distance $d_{g}+d_{\mathrm{m}}$, where $d_{\mathrm{m}}$ is a transmission decision margin that is used to guarantee the protection of the PUs when the SB actually resides in the extended $\mathrm{PN}$ coverage, i.e. $d_{\mathrm{SU}_{0}} \leq d_{g}$. The protection is guaranteed by setting $d_{\mathrm{m}}$ in such a way that the transmission within the extended PN coverage is allowed with a probability equal or less than the CTP, $\dot{\mathrm{P}}_{C}$. The validity of protection by the $d_{\mathrm{m}}$ will be shown in Fig. 4 . The result of this is a binary transmission decision variable, $D_{T}$, where $D_{T}=1$ indicates that the transmission is allowed, and $\mathrm{D}_{T}=0$ rejects it. If transmission is allowed, the SB continues to the next step.

In the second step, the SB applies a transmit power margin $T_{\mathrm{m}}$ that guarantees the PU protection when the SB resides outside the extended $\mathrm{PN}$ coverage, i.e., $d_{\mathrm{SU}_{0}}>d_{g}$.

The transmit power margin $T_{\mathrm{m}}$ depends on the locations of the PU receiver and the SB corresponding to the transmitter. Since the exact location information is not available at the $\mathrm{SB}$, we set $T_{\mathrm{m}}$ considering the worst case in which the PU receiver lies in the neighborhood of edge of the extended PN coverage and the SB is at a location leading to maximum $T_{\mathrm{m}}$. This fact will be confirmed in Fig. 5.

\subsection{Distance estimation in MATP-I and MATP-C}

From (1) and (2), $d_{\mathrm{SU}_{0}}$ can be estimated with

$$
\hat{d}_{\mathrm{SU}_{0}}^{(Z)}=d_{0} 10^{-\left(\hat{R}^{(Z)}+T_{\mathrm{PU}_{0}}-20 \log _{10}\left(4 \pi d_{0} / \lambda\right)\right) /(10 \eta)},
$$

where the superscript $(Z)$ indicates type of MATP, i.e., $(P)$ for MATP-P, $(C)$ for MATP-C, and $(I)$ for MATP-I, and $\hat{R}^{(Z)}$ denotes estimated $\bar{R}_{\mathrm{PU}_{0} \rightarrow \mathrm{SU}_{0}} . \hat{R}^{(Z)}$ for MATP-I and MATP-C are

$$
\begin{aligned}
\hat{R}^{(I)} & =R_{\mathrm{PU}_{0} \rightarrow \mathrm{SU}_{0}}, \\
\hat{R}^{(C)} & =\frac{\sum_{n=0}^{N_{S}} R_{\mathrm{PU}_{0} \rightarrow \mathrm{SU}_{n}}}{1+N_{s}},
\end{aligned}
$$

respectively. In the case of MATP-C in (8), RSS values from different SUs are averaged in order to suppress the effect of shadowing [40]. In $\hat{R}^{(C)}, N_{S}$ can be interpreted as the number of estimations by SUs at different locations. Specifically, the location of SB is fixed; however, mobile SUs can obtain $R_{\mathrm{PU}_{0} \rightarrow \mathrm{SU}_{n}}$ at the different locations. The estimation $\hat{d}_{\mathrm{SU}_{0}}^{(I)}$ corresponds to a maximum likelihood estimation [41].

\subsection{Transmission decision with transmission decision margin}

The transmission decision rule is defined as

$$
\mathrm{D}_{T}= \begin{cases}1 ; & \left(\hat{d}_{\mathrm{SU}_{0}}^{(Z)} \geq d_{g}+d_{m}^{(Z)}\right) \\ 0 ;\left(\hat{d}_{\mathrm{SU}_{0}}^{(Z)}<d_{g}+d_{m}^{(Z)}\right)\end{cases}
$$

The transmission decision margin $d_{\mathrm{m}}^{(Z)}$ is set to satisfy the equality

$$
\operatorname{Pr}\left(\hat{d}_{\mathrm{SU}_{0}}^{(Z)} \geq d_{g}+d_{\mathrm{m}}^{(Z)} \mid d_{\mathrm{SU}_{0}}=d_{g}\right)=\dot{\mathrm{P}}_{C}
$$

This shows that the probability of allowing transmission when the SB is within the extended PN coverage is always less than or equal to the CTP, i.e., $\operatorname{Pr}\left(\mathrm{D}_{T}=1 \mid d_{\mathrm{SU}_{0}} \leq\right.$ $\left.d_{g}\right) \leq \dot{\mathrm{P}}_{C}$. The transmission decision margin, $d_{\mathrm{m}}^{(Z)}$, is derived in Section 4.2. 


\subsection{Maximum allowable transmit power setting based on distance estimation}

In the MATP-I and the MATP-C, we use a transmit power margin $T_{\mathrm{m}}^{(Z)}$ to satisfy the constraint in the region where $d_{\mathrm{SU}_{0}}>d_{g}$. According to the transmission decision, the MATP is given by

$T_{\mathrm{SU}_{0}, \max }^{(Z)}= \begin{cases}I_{\mathrm{th}}+L\left(\hat{d}_{\mathrm{SU}_{0}}^{(Z)}-r_{c, p}\right)-\sigma_{x} Q^{-1} & \left(\dot{\mathrm{P}}_{\mathrm{C}}\right)-T_{\mathrm{m}}^{(Z)} \\ \text { No transmission; } & \left(\mathrm{D}_{T}=1\right) \\ & (11)\end{cases}$

The transmit power margin, $T_{\mathrm{m}}^{(Z)}$, is derived in Section 4.3.

\section{Analysis}

In this section, a probability density function (PDF) for the distance estimations by the estimator in (6) is shown. The PDF is used to derive the transmission decision margin $d_{\mathrm{m}}^{(Z)}$ which satisfies (10) and is used in the transmission decision rule (9). Finally, we derive the transmit power margin $T_{\mathrm{m}}^{(Z)}$ used in the MATP-I or the MATP-C schemes, to satisfy (11).

\subsection{Analysis of distance estimation}

The SUs are assumed to be located uniformly over the disk corresponding to the SB coverage area. The conditional PDF of the $d_{\mathrm{SU}_{n}}$ (MATP-C) given that $d_{\mathrm{SU}_{0}}=d$ is $p_{d_{\mathrm{SU}_{n}}}\left(d_{\mathrm{SU}_{n}} \mid d_{\mathrm{SU}_{0}}=d\right)$. For simplicity with slight abuse of the notation, we denote this as $p_{d_{\mathrm{SU}_{n}}}\left(d_{\mathrm{SU}_{n}} \mid d_{\mathrm{SU}_{0}}\right)$. It can written as

$p_{d_{\mathrm{SU}_{n}}}\left(d_{\mathrm{SU}_{n}} \mid d_{\mathrm{SU}_{0}}\right)=\frac{2 d_{\mathrm{SU}_{n}}}{\pi r_{c, s}^{2}} \cos ^{-1}\left(\frac{d_{\mathrm{SU}_{n}}^{2}+d_{\mathrm{SU}_{0}}^{2}-r_{c, s}^{2}}{2 d_{\mathrm{SU}_{n}} d_{\mathrm{SU}_{0}}}\right)$

Note that the condition does not mean that $d_{\mathrm{SU}_{0}}$ is available at the $\mathrm{SN}$.

A conditional PDF of RSS without shadowing effects $\left(\bar{R}_{\mathrm{PU}_{0} \rightarrow \mathrm{SU}_{n}}\right)$ can be derived through a transformation of the PDF $p\left(d_{\mathrm{SU}_{n}} \mid d_{\mathrm{SU}_{0}}\right)$ with a function $\bar{R}_{\mathrm{PU}_{0} \rightarrow \mathrm{SU}_{n}}=T_{\mathrm{PU}_{0}}-$ $L\left(d_{\mathrm{SU}_{n}}\right)$ and (2) resulting in

$$
\begin{aligned}
& p_{\bar{R}_{\mathrm{PU}_{0} \rightarrow \mathrm{SU}}}\left(\bar{R}_{\mathrm{PU}_{0} \rightarrow \mathrm{SU}_{n}} \mid d_{\mathrm{SU}_{0}}\right)= \\
& \frac{2 C_{1} C_{2}^{\bar{R}_{\mathrm{PU}_{0} \rightarrow \mathrm{SU}_{n}}}\left|C_{1} C_{2}^{\bar{R}_{\mathrm{PU}_{0} \rightarrow \mathrm{SU}_{n}}} \ln C_{2}\right|}{\pi r_{c, s}^{2}} . \\
& \cos ^{-1}\left(\frac{\left(C_{1} C_{2}^{\bar{R}_{\mathrm{PU}_{0} \rightarrow \mathrm{SU}_{n}}}\right)^{2}+d_{\mathrm{SU}_{0}}^{2}-r_{c, s}^{2}}{2 C_{1} C_{2}^{\bar{P}_{\mathrm{PU}_{0} \rightarrow \mathrm{SU}_{n}}} d_{\mathrm{SU}_{0}}}\right),
\end{aligned}
$$

where

$$
\begin{aligned}
& C_{1}=d_{0} 10\left(T_{\mathrm{PU}_{0}}-10 \log _{10}\left(\frac{4 \pi d_{0}}{\lambda}\right)^{2}\right) /(10 \eta) \\
& C_{2}=10^{-1 /(10 \eta)} .
\end{aligned}
$$

The conditional PDF of the RSS including shadowing effects $\left(R_{\mathrm{PU}_{0} \rightarrow \mathrm{SU}_{n}}\right)$ is obtained with

$$
\begin{aligned}
& p_{R_{\mathrm{PU}_{0} \rightarrow \mathrm{SU}_{n}}}\left(R_{\mathrm{PU}_{0} \rightarrow \mathrm{SU}_{n}} \mid d_{\mathrm{SU}_{0}}\right)= \\
& \int_{\bar{R}_{\mathrm{PU}_{0} \rightarrow \mathrm{SU}_{n}}=-\infty}^{\bar{R}_{\mathrm{PU}_{0} \rightarrow \mathrm{SU}_{n}}=\infty} \frac{1}{\sqrt{2 \pi} \sigma_{x}} \exp \left(\frac{-\left(R_{\mathrm{PU}_{0} \rightarrow \mathrm{SU}_{n}}-\bar{R}_{\mathrm{PU}_{0} \rightarrow \mathrm{SU}_{n}}\right)^{2}}{2 \sigma_{x}^{2}}\right) \\
& \cdot p_{\bar{R}_{\mathrm{PU}_{0} \rightarrow \mathrm{SU}_{n}}}\left(\bar{R}_{\mathrm{PU}_{0} \rightarrow \mathrm{SU}_{n}} \mid d_{\mathrm{SU}_{0}}\right) d \bar{R}_{\mathrm{PU}_{0} \rightarrow \mathrm{SU}_{n}} .
\end{aligned}
$$

In the case of MATP-I, the mean and variance of $\hat{R}^{(I)}$ defined in (8) are given by

$$
\mu_{\hat{R}}^{(I)}=T_{\mathrm{PU}_{0}}-L\left(d_{\mathrm{SU}_{0}}\right),
$$

and

$$
v_{\hat{R}}^{(I)}=\sigma_{x}^{2} .
$$

On the other hand, in the case of MATP-C, according to (8) and (14), the mean and variance of $\hat{R}^{(C)}$ defined in (7) are given by

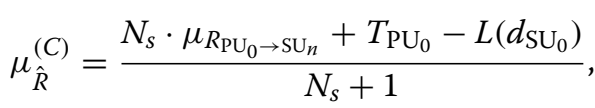

and

$$
v_{\hat{R}}^{(C)}=\frac{N_{s} \cdot v_{R_{\mathrm{PU}_{0} \rightarrow \mathrm{SU}_{n}}}+\sigma_{x}^{2}}{\left(N_{s}+1\right)^{2}} .
$$

In (17) and (18), $\mu_{R_{\mathrm{PU}_{0} \rightarrow \mathrm{SU}}}$ and $\nu_{R_{\mathrm{PU}_{0} \rightarrow \mathrm{SU}_{n}}}$ correspond to the mean and variance of $R_{\mathrm{PU}_{0} \rightarrow \mathrm{SU}_{n}}$ in (14), respectively, and thus they are available by numerical calculations.

Using Gaussian approximation, $p_{\hat{R}^{(Z)}}\left(\hat{R}^{(Z)} \mid d_{\mathrm{SU}_{0}}\right)$ is given by

$p_{\hat{R}^{(Z)}}\left(\hat{R}^{(Z)} \mid d_{\mathrm{SU}_{0}}\right)=\frac{1}{\sqrt{2 \pi v_{\hat{R}}^{(Z)}}} \exp \left(\frac{-\left(\hat{R}^{(Z)}-\mu_{\hat{R}}^{(Z)}\right)^{2}}{2 v_{\hat{R}}^{(Z)}}\right)$

Finally, the PDF of $\hat{d}_{\mathrm{SU}_{0}}^{(Z)}$, given $d_{\mathrm{SU}_{0}}$, is

$$
\begin{gathered}
p_{\hat{d}_{\mathrm{SU}_{0}}^{(Z)}}\left(\hat{d}_{\mathrm{SU}_{0}}^{(Z)} \mid d_{\mathrm{SU}_{0}}\right)=\frac{1}{\left|\hat{d}_{\mathrm{SU}_{0}}^{(Z)} \ln C_{2}\right| \sqrt{2 \pi v_{\hat{R}}^{(Z)}}} . \\
\exp \left(\frac{-\left(\ln \left(\hat{d}_{\mathrm{SU}_{0}}^{(Z)} / C_{1}\right) / \ln C_{2}-\mu_{\hat{R}}^{(Z)}\right)^{2}}{2 v_{\hat{R}}^{(Z)}}\right) .
\end{gathered}
$$

For verification of the analysis, $p\left(\hat{d}_{\mathrm{SU}_{0}}^{(C)} \mid d_{\mathrm{SU}_{0}}\right)$ : for MATP-C and $p\left(\hat{d}_{\mathrm{SU}_{0}}^{(I)} \mid d_{\mathrm{SU}_{0}}\right)$ : for MATP-I are plotted in Fig. 3. The analyses in Fig. 3 are obtained by (20). The simulation parameters are $\eta=3, \sigma_{x}=9 \mathrm{~dB}$, and $d_{\mathrm{SU}_{0}}=20 \mathrm{~km}$. For MATP-C, two results with $N_{S}=4$ and $N_{S}=8$ are plotted. The simulation validates our analysis. Figure 3 also shows that increasing $N_{S}$ can achieve smaller variance of 


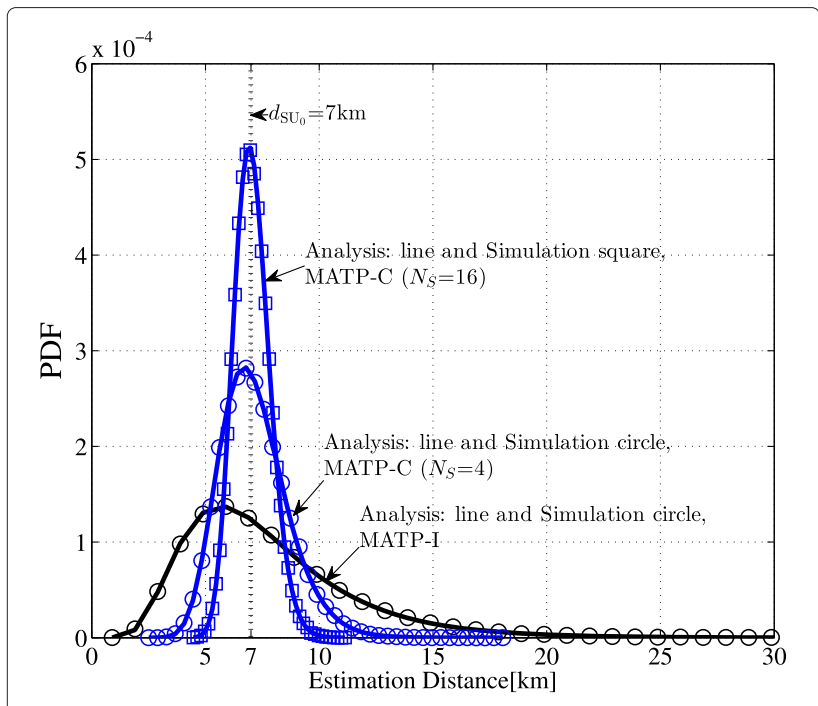

Fig. 3 The PDFs of distance estimates for the MATP-C $\left(N_{S}=4\right)$ and the MATP-I, from analysis (line) and simulation (circle), for $N_{S}=4, \eta=3$, $\sigma_{x}=9 \mathrm{~dB}$, and $r_{c, S}=500 \mathrm{~m}$, the actual distance is $d_{S U_{0}}=20 \mathrm{~km}$

estimates in the MATP-C. That is to say that the averaging employed by MATP-C achieves a more accurate estimation similar to [40].

\subsection{Transmission decision margin setting based on analysis}

The proper $d_{\mathrm{m}}^{(Z)}$ is given by

$$
d_{\mathrm{m}}^{(Z)}=f_{\hat{d}_{\mathrm{SU}_{0}}^{(\mathrm{Z})}}^{-1}\left(\dot{\mathrm{P}}_{C} \mid d_{\mathrm{SU}_{0}}=d_{g}\right)-d_{g}
$$

where $f_{\hat{d}_{\mathrm{SU}_{0}}^{(Z)}}$ is the cumulative distribution function (CDF) corresponding to the PDF in (20).

In Fig. 4, the transmission probability, $\operatorname{Pr}\left(D_{T}=1\right)$ is plotted as a function of $d_{\mathrm{SU}_{0}}$ in terms of the MATP-I and MATP-C with varying $\sigma_{x}(3$ and $9 \mathrm{~dB})$. The margin $d_{\mathrm{m}}^{(Z)}$ which is set according to $(21)$ is found by numerical techniques. The result verifies that $d_{\mathrm{m}}^{(Z)}$ can satisfy $\operatorname{Pr}\left(\mathrm{D}_{T}=1\right)=\dot{\mathrm{P}}_{C}$ when $d_{\mathrm{SU}_{0}}=d_{g}=4.2 \mathrm{~km}$. This also means that in the region $d_{\mathrm{SU}_{0}} \leq d_{g}, \operatorname{Pr}\left(\mathrm{D}_{T}=1\right) \leq$ $\dot{\mathrm{P}}_{\mathrm{C}}$. Thus, $\mathrm{P}_{\mathrm{C}} \leq \dot{\mathrm{P}}_{\mathrm{C}}$ in the region $\bar{d}_{\mathrm{SU}} \leq d_{g}$. The rate of increase of the transmission probability $\operatorname{Pr}\left(\mathrm{D}_{T}=1\right)$ depends on $\sigma_{x}$. Specifically, a smaller $\sigma_{x}$ achieves a larger rate of increase. In addition, in the region $d_{\mathrm{SU}_{0}} \geq d_{g}$, the MATP-C can achieve a higher $\operatorname{Pr}\left(\mathrm{D}_{T}=1\right)$ than the MATP-I. This observation implies that the MATP-C has greater potential to achieve a more effective spectrum utilization.

\subsection{Transmit power margin setting based on analysis}

We obtain the transmit power margin under a given $d_{\mathrm{SU}_{0}}$. Since the exact distance is unknown, the SB uses the $d_{\mathrm{SU}_{0}}$ leading to the maximum (worst-case) margin.

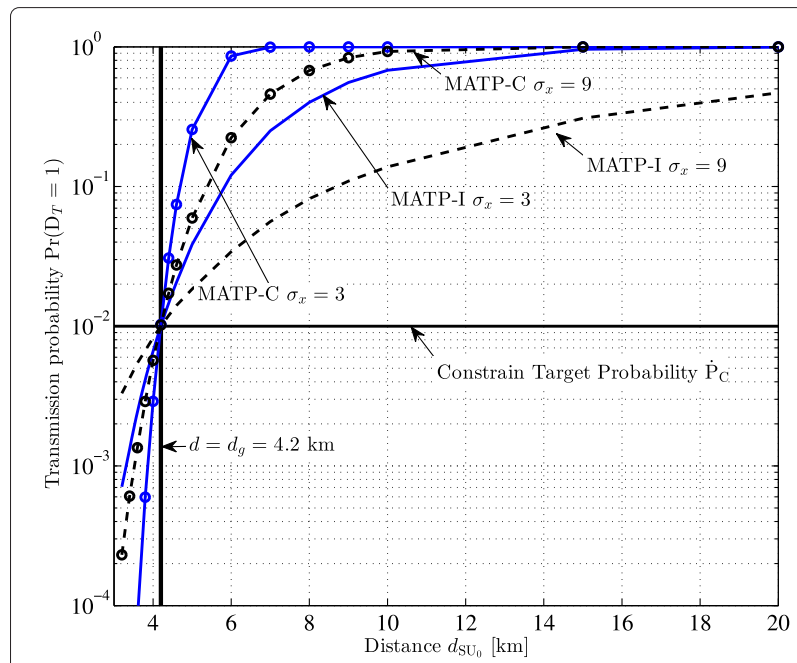

Fig. 4 Probability of transmission $\left(\operatorname{Pr}\left(D_{T}=1\right)\right)$ as a function of $d_{S U_{0}}$ for the MATP-C $\left(N_{S}=4\right.$, solid line) and the MATP-I (dashed line), for $N_{S}=4, \eta=3, \sigma_{X}=3$ and $9 \mathrm{~dB}, d_{g}=4.2 \mathrm{~km}, r_{C, S}=500 \mathrm{~m}$, and $\dot{\mathrm{P}}_{\mathrm{C}}=0.01$

In the case $\mathrm{D}_{T}=1$, the minimum $\hat{d}_{\mathrm{SU}_{0}}^{(Z)}$ is equal to $d_{g}+$ $d_{\mathrm{m}}^{(Z)}$. Thus, the smallest possible MATP value given by (11) is

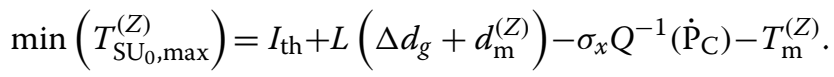

A conditional PDF of the MATP $T_{\mathrm{SU}_{0}, \max }^{(Z)}$ in (11) given $d_{\mathrm{SU}_{0}}$ is obtained by univariate transformation from estimated distance $\hat{d}_{\mathrm{SU}_{0}}$ in (20) to $T_{\mathrm{SU}_{0}, \max }^{(Z)}$ and is given by

$$
\begin{aligned}
& p_{T_{\mathrm{SU}_{0}, \max }^{(\mathrm{Z})}}\left(T_{\mathrm{SU}_{0}, \max }^{(\mathrm{Z})} \mid d_{\mathrm{SU}_{0}}\right)=
\end{aligned}
$$

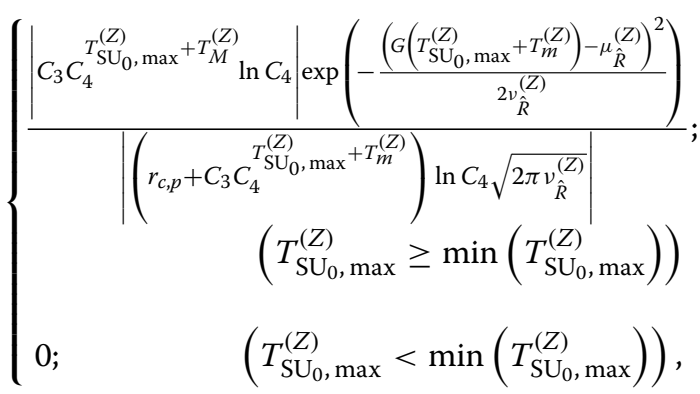

where $C_{3}, C_{4}$, and $D\left(T_{\mathrm{SU}_{0}, \max }^{(Z)}+T_{m}^{(Z)}\right)$ are

$$
\begin{aligned}
C_{3} & =d_{0} 10\left(-I_{\mathrm{th}}-10 \log _{10}\left(\frac{4 \pi d_{0}}{\lambda}\right)^{2}+\sigma_{x} Q^{-1}\left(\dot{\mathrm{P}}_{\mathrm{C}}\right)\right) /(10 \eta) \\
C_{4} & =1 / C_{2} \\
G(x) & =\frac{\ln \left(\frac{r_{c, p}+C_{3} C_{4}^{x}}{C_{1}}\right)}{\ln C_{2}} .
\end{aligned}
$$


The condition $\left(T_{\mathrm{SU}_{0}, \max }^{(\mathrm{Z})}<\min \left(T_{\mathrm{SU}_{0}, \max }^{(Z)}\right)\right)$ in indicates that $\mathrm{D}_{T}=0$.

Due to the shadowing effect from the $\mathrm{PB}$ to the $\mathrm{SN}$ and random locations of the SUs in the case of the MATP-C, $T_{\mathrm{SU}_{0}, \max }^{(\mathrm{Z})}$ is random. From (2) and (3), the interference level at the PU determined by path loss, $\bar{I}_{\mathrm{PU}}$, can be expressed as

$$
\bar{I}_{\mathrm{PU}}=T_{\mathrm{SU}_{0}, \max }^{(Z)}-C_{5}
$$

where

$$
C_{5}=\left(10 \log _{10}\left(\frac{4 \pi d_{0}}{\lambda}\right)^{2}+10 \eta \log _{10}\left(\frac{d_{\mathrm{SU}_{0}}-r_{c, p}}{d_{0}}\right)\right) \text {. }
$$

Since $d_{\mathrm{SU}_{0}}$ is given, the minimum interference level, $\min \left(\bar{I}_{\mathrm{PU}}\right)$, is found by substituting $\min \left(T_{\mathrm{SU}_{0}, \max }^{\left(\mathrm{Z}_{\mathrm{C}}\right.}\right)$ into (24). The conditional PDF $p_{\bar{I}_{\mathrm{PU}}}\left(\bar{I}_{\mathrm{PU}} \mid d_{\mathrm{SU}_{0}}\right)$ is determined through a univariate transformation from $T_{\mathrm{SU}_{0}, \max }^{(Z)}$ to $\bar{I}_{\mathrm{PU}}$ and results in

$$
\begin{aligned}
& p_{\bar{I}_{\mathrm{PU}}}\left(\bar{I}_{\mathrm{PU}} \mid d_{\mathrm{SU}_{0}}\right)= \\
& \left\{\begin{aligned}
&\left|C_{3} C_{4}^{\bar{I}_{\mathrm{PU}}+C_{5}+T_{M}^{(Z)}} \ln C_{4}\right| \exp \left(-\frac{\left(G\left(\bar{I}_{\mathrm{PU}}+C_{5}+T_{M}^{(Z)}\right)-\mu_{\hat{\mathrm{R}}}^{(Z)}\right)^{2}}{2 v_{\hat{R}}^{(Z)}}\right) \\
&\left(r_{c, p}+C_{3} C_{4}^{\bar{I}_{\mathrm{PU}}+C_{5}+T_{M}^{(Z)}}\right) \\
& \ln C_{4} \sqrt{2 \pi v_{\hat{R}}^{(Z)}} \\
&\left(\bar{I}_{\mathrm{PU}}>\min \left(\bar{I}_{\mathrm{PU}}\right)\right) \\
& 0 ;\left(\bar{I}_{\mathrm{PU}} \leq \min \left(\bar{I}_{\mathrm{PU}}\right)\right) .
\end{aligned}\right.
\end{aligned}
$$

A conditional PDF of the interference level including the log-normal shadowing effects from the PB to the SUs and from the SB to the PU is found from (3) and (25) and is given by

$$
\begin{aligned}
& p_{I_{\mathrm{PU}}}\left(I_{\mathrm{PU}} \mid d_{\mathrm{SU}_{0}}\right)=\int_{\bar{I}_{\mathrm{PU}}=\min \left(\bar{I}_{\mathrm{PU}}\right)}^{\bar{I}_{\mathrm{PU}}=\infty} p_{I_{\mathrm{PU}}}\left(I_{\mathrm{PU}} \mid d_{\mathrm{SU}_{0}}, \bar{I}_{\mathrm{PU}}\right) . \\
& p_{\bar{I}_{\mathrm{PU}}}\left(\bar{I}_{\mathrm{PU}} \mid d_{\mathrm{SU}_{0}}\right) d \bar{I}_{\mathrm{PU}},
\end{aligned}
$$

where

$$
p_{I_{\mathrm{PU}}}\left(I_{\mathrm{PU}} \mid d_{\mathrm{SU}}, \bar{I}_{\mathrm{PU}}\right)=\frac{1}{\sqrt{2 \pi} \sigma_{x}} \exp \left(-\frac{\left(I_{\mathrm{PU}}-\bar{I}_{\mathrm{PU}}\right)^{2}}{2 \sigma_{x}^{2}}\right)
$$

and represents the log-normal shadowing effect from the SB to the PU. The shadowing effect from PB to SUs was similarly represented by the first term inside the integral in (14) and is already included in (25).

The constraint probability as a function of the margin $T_{\mathrm{m}}^{(Z)}$ for a given $d_{\mathrm{SU}_{0}}$ is given by

$\mathrm{P}_{\mathrm{C}}\left(T_{m}^{(Z)} \mid d_{\mathrm{SU}_{0}}\right)=\int_{I_{\mathrm{PU}}=I_{\mathrm{th}}}^{I_{\mathrm{PU}}=\infty} p_{I_{\mathrm{PU}}}\left(I_{\mathrm{PU}} ; T_{m}^{(Z)} \mid d_{\mathrm{SU}_{0}}\right) d I_{\mathrm{PU}}$
The satisfaction of the constraint probability requires that $\mathrm{P}_{\mathrm{C}} \leq \dot{\mathrm{P}}_{\mathrm{C}}$. Equation (28) implies that the required margin $T_{\mathrm{m}}^{(Z)}$ depends on the distance $d_{\mathrm{SU}_{0}}$ as

$$
T_{\mathrm{m}}^{(Z)}=\mathrm{P}_{\mathrm{C}}^{-1}\left(\dot{\mathrm{P}}_{\mathrm{C}} \mid d_{\mathrm{SU}_{0}}\right) \text {. }
$$

Thus, we consider the worst-case $d_{\mathrm{SU}_{0}}$ when setting the margin. This can be expressed as

$$
T_{m}^{(Z) *}=\max _{d_{g}<d_{\mathrm{SU}_{0}<\infty}} \mathrm{P}_{\mathrm{C}}^{-1}\left(\dot{\mathrm{P}}_{\mathrm{C}} \mid d_{\mathrm{SU}_{0}}\right) .
$$

The transmission decision margin $d_{\mathrm{m}}$ influences the $T_{\mathrm{SU}_{0}, \min }^{(Z)}$ as (22), and this indicates that $d_{\mathrm{m}}$ affects the worst-case $T_{\mathrm{m}}^{(\mathrm{Z}) *}$.

To see the worst case for $T_{\mathrm{m}}^{(Z)}$ (i.e., $\left.T_{\mathrm{m}}^{(Z) *}\right), T_{\mathrm{m}}^{(Z)}$ satisfying $\dot{\mathrm{P}}_{\mathrm{C}}$ as a function of $d_{\mathrm{SU}_{0}}$ in the cases of MATP-I and the MATP-C is shown in Fig. 5. The parameters are set as $N_{S}=4, \eta=3, \sigma_{x}=9 \mathrm{~dB}, r_{c, p}=3.68 \mathrm{~km}$, and $r_{c, s}=500 \mathrm{~m}$. Values for $d_{g}$ of 3.7 and $4.2 \mathrm{~km}$ are used.

In the region where $d_{\mathrm{SU}_{0}}<d_{g}$, the margin $d_{\mathrm{m}}$ is used in the transmission decision variable $\mathrm{D}_{T}$, to satisfy the constraint. That is, $\operatorname{Pr}\left(\mathrm{D}_{T}=1\right) \leq \dot{\mathrm{P}}_{\mathrm{C}}$, therefore $T_{\mathrm{m}}^{(Z)}=0$. The maximal values are in the region where $d_{\mathrm{SU}_{0}} \geq d_{g}$ and close to $d_{\mathrm{SU}_{0}}=d_{g}$. In this region, $T_{\mathrm{m}}^{(Z)}$ increases at a rapid rate. This is because $\operatorname{Pr}\left(\mathrm{D}_{T}=1\right)$ increases, and to satisfy the constraint, a larger $T_{\mathrm{m}}^{(Z)}$ is required. In the region to the right of the maximum, $T_{\mathrm{m}}^{(Z)}$ decreases slowly since $\operatorname{Pr}\left(\mathrm{D}_{T}=1\right) \gg \dot{\mathrm{P}}_{\mathrm{C}}$ and the far $\mathrm{SB}$ transmitter requires smaller transmit power margin. Since the SB does not have exact information about its location $d_{\mathrm{SU}_{0}}$, we set the transmit power margin to the maximal value, $T_{\mathrm{m}}^{(Z) *}$.

In the case when $d_{g}=3.7 \mathrm{~km}$, the SB may be located near the PU receiver, and thus it requires a significantly

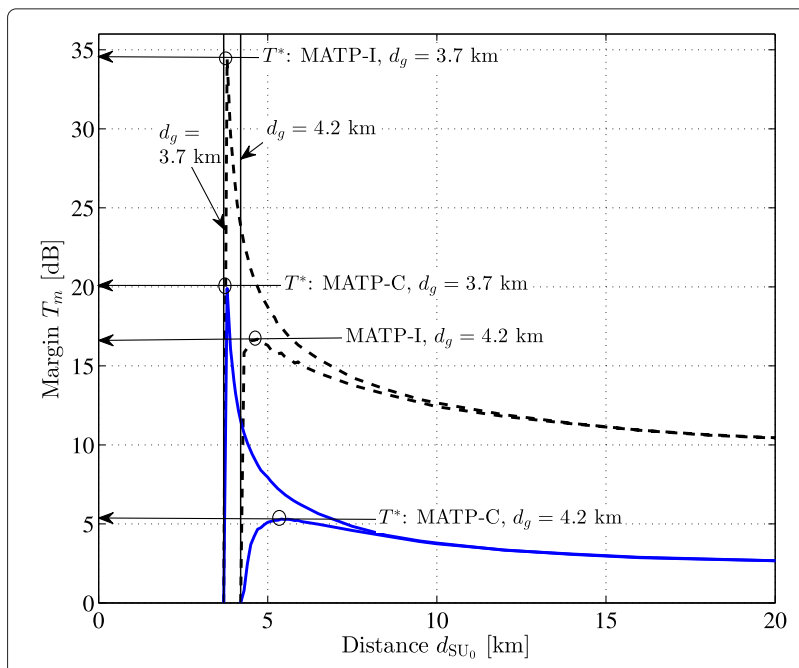

Fig. 5 The required margin $T_{m}$ (29) as a function of $d_{S U_{0}}$ for MATP-C $\left(N_{S}=4\right)$, and MATP-I, with $\dot{P}_{C}=0.01, N_{S}=4, \eta=3, \sigma_{x}=9 \mathrm{~dB}$, $r_{c, S}=500 \mathrm{~m}, r_{c, p}=3.68 \mathrm{~km}, d_{g}=3.7$, and $4.2 \mathrm{~km}$ 
large margin. For example, in the case of MATP-I, $T_{\mathrm{m}}^{(\mathrm{Z}) *}=$ $34 \mathrm{dBm}$. On the other hand, in the case of MATP-C with $d_{g}=4.2 \mathrm{~km}$, the required margin is only $T_{\mathrm{m}}^{(Z) *}=5 \mathrm{dBm}$. The difference between these margins is $29 \mathrm{dBm}$ and is caused by not only the gain of the cooperative measurement but also by the appropriate $d_{g}$ setting.

In Fig. $6, T_{\mathrm{m}}^{*}$ as a function of $d_{g}$ in terms of MATP-C and the MATP-I is shown. This result shows that smaller $d_{g}$ values require significantly large margins. In the case of MATP-I, the difference between $d_{g}=3.7 \mathrm{~km}$ and $T_{\mathrm{m}}^{*}$ at $d_{g}=4.2 \mathrm{~km}$ is $17 \mathrm{dBm}$, and in the case of MATP-C, the difference is still $15 \mathrm{dBm}$.

\section{Numerical results}

In this section, MATP-P, MATP-I, and MATP-C are compared in terms of the average capacity, $\bar{C}_{\text {down }}$ similarly as in $[28,29]$. In fact, the MATP-P is equivalent to the approach proposed in $[28,29]$ where the perfect location information is assumed to be available and a comparison with the MATP-P corresponds to a comparison with existing method. A derivation of the average capacity is shown in the following subsection.

The CTP is set to $\dot{\mathrm{P}}_{\mathrm{C}}=0.01$. The assumed center frequency of the spectrum band is $600 \mathrm{MHz}$, which is used in digital TV broadcasting, but the application of the proposed method is not limited to it. We set the path loss exponent as $\eta=3$. The transmit power of the $\mathrm{PB}$ is set to $T_{\mathrm{PU}_{0}}=60 \mathrm{dBm}$, and the total transmit power of the $\mathrm{SB}$ is always limited to the maximum value of $T_{\text {total }}=30 \mathrm{dBm}$.

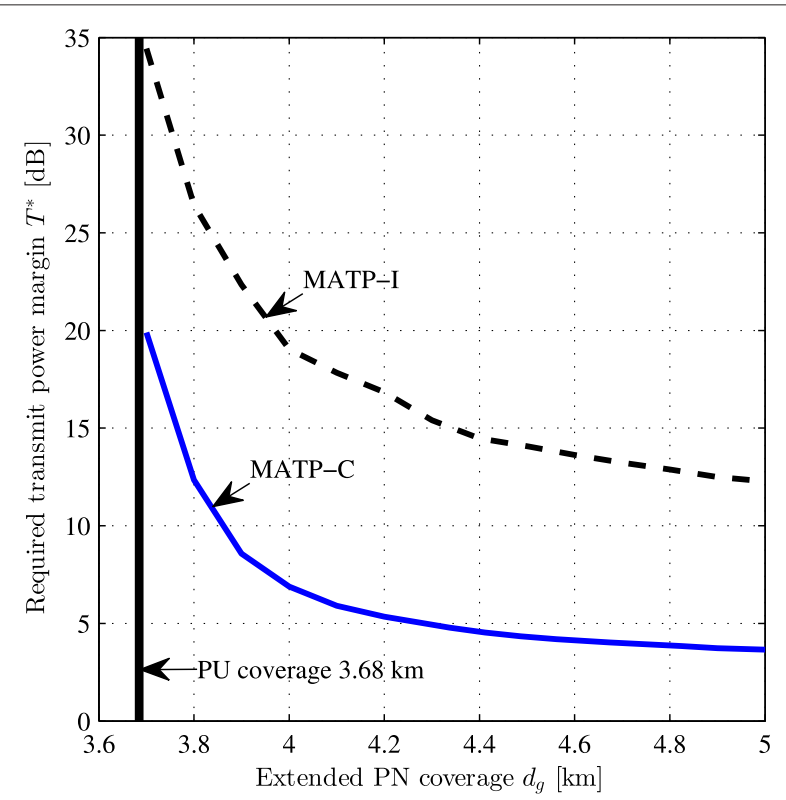

Fig. 6 The required transmit power margin $T_{\mathrm{m}}^{*}$ as a function of extended PN coverage $d_{g}$ for MATP-C $\left(N_{S}=4\right)$ and MATP-I, with $\dot{P}_{C}=0.01, \eta=3, \sigma_{x}=9 \mathrm{~dB}, r_{C, P}=3.68 \mathrm{~km}$, and $r_{C, S}=500 \mathrm{~m}$
The minimum required received signal levels, $\gamma_{\mathrm{PN}}$ and $\gamma_{\mathrm{SN}}$, are set as $-75 \mathrm{dBm}$ and $-85 \mathrm{~dB}$, respectively, leading to the radii $r_{c, p}=3.68$ and $r_{c, s}=0.5 \mathrm{~km}$.

\subsection{Average capacity obtained by power and channel allocation}

The SB allocates a transmit power $T_{n, l, \mathrm{~mW}}$ for the $n$th SU transmission on the $l$ th sub-channel, where the bandwidth $B$ is divided into $L$ sub-channels. Note that in this paper, we use two units, "mW" and "dBm" for variables corresponding to power values. When unit in a variable is $\mathrm{mW}$, "mW" is noted in the suffix (for example, $T_{n, l, \mathrm{~mW}}$ ), but when the unit is $\mathrm{dBm}$, notation of unit is abbreviated (for example, $T_{n, l}$ ). The aim of this resource allocation is to maximize the downlink capacity $C_{\text {down }}$ while keeping the interference constraint and a total transmit power constraint. This is expressed as

$$
\begin{aligned}
\max C_{\text {down }} & =\max \frac{1}{L} \sum_{i=1}^{N_{S}} \sum_{l=1}^{L} \\
& a_{n, l} \log _{2}\left(1+\frac{\left|h_{n, l, \mathrm{SB}}\right|^{2} T_{n, l, \mathrm{~mW}}}{N_{\mathrm{mW}}+\left|h_{n, l, \mathrm{~PB}}\right|^{2} T_{\mathrm{PU}_{0}, \mathrm{~mW}} / L}\right)
\end{aligned}
$$

subject to

$$
\begin{aligned}
& \sum_{n=1}^{N_{s}} a_{n, l} \leq 1, \forall l, a_{n, l} \in\{0,1\} \forall n, l, \\
& \sum_{n=1}^{N_{s}} \sum_{l=1}^{L} a_{i, l} T_{n, l, \mathrm{~mW}} \leq T_{\text {total, } \mathrm{mW}}, \\
& \sum_{n=1}^{N_{s}} \sum_{l=1}^{L} a_{i, l} T_{n, l, \mathrm{~mW}} \leq T_{\mathrm{SU}_{0}, \mathrm{max}, \mathrm{mW}}^{(\mathrm{Z})},
\end{aligned}
$$

where $a_{n, l}$ is a sub-channel allocation indicator (i.e., $a_{n, l}=$ 1 indicates that the $l$ th sub-channel is allocated to the $n$th SU transmission; otherwise, $\left.a_{n, l}=0\right) ; h_{n, l, \mathrm{~PB}}$ denotes the channel gain between the PB and the $n$th $\mathrm{SU}$ for $l$ th subchannel; and $N_{\mathrm{mW}}$ is the noise power in one sub-channel. In the capacity, the interference from the $\mathrm{PB}$ which is assumed to be divided equally into all sub-channels is considered.

The constraints are as follows: (32) indicates that each sub-channel is assigned to only one SU, (33) is the total transmit power constraint due to SB limitations or constraints by a regulator, and (34) is the interference constraint.

Without loss of generality, we use capacity normalized by sub-channel bandwidth and $L$ in (31). The solution of this optimization problem can be found by a simple waterfilling scheme as described in [28, 42].

The average $C_{\text {down }}$ is given by $\bar{C}_{\text {down }}=E\left[C_{\text {down }}\right]$, where $E[\cdot]$ denotes the expectation function calculated 
with respect to channel gains and locations of SUs. Specifically, the locations of SUs in the SB's coverage area are changed according to a uniform distribution to calculate $\bar{C}_{\text {down }}$ while the channel gains $\left|h_{n, l, \mathrm{SB}}\right|$ and $\left|h_{n, l, \mathrm{~PB}}\right|$ are due to distance between the transmitter and receiver, the shadowing, and Rayleigh fading. In addition, we assume that the noise levels per sub-channel in the SUs are assumed to be the same, and $N$ is $-95 \mathrm{dBm}$.

\subsection{Average capacity performances of MATPs}

In Figs. 7 and $8, \bar{C}_{\text {down }}$ as a function of $d_{\mathrm{SU}_{0}}$ is shown. In Fig. $7, \sigma_{x}=3 \mathrm{~dB}$ while in Fig. $8, \sigma_{x}=9 \mathrm{~dB}$. The $\bar{C}_{\text {down }}$ are increasing functions in terms of $d_{\mathrm{SU}_{0}}$ since MATP increases as $d_{\mathrm{SU}_{0}}$ increases. We can confirm that MATP-C always outperforms MATP-I in these results since MATP$\mathrm{C}$ requires reduced margin, as shown previously by Fig. 5 .

In Fig. 7, MATP-C and the MATP-I achieve the same performance as MATP-P in the regions $d_{\mathrm{SU}_{0}}>7$ and $d_{\mathrm{SU}_{0}}>14 \mathrm{~km}$, respectively. This shows that the probability of transmission $\left(\operatorname{Pr}\left(\mathrm{D}_{T}=1\right)\right)$ of MATP-C and MATP-I approaches 1, as confirmed in Fig. 4. It also shows that $T_{\text {total }}<T_{\mathrm{SU}_{0}, \max }^{(Z)}$, so that MATP setting does not anymore limit the transmit power.

In the case of $\sigma_{x}=9 \mathrm{~dB}$ (Fig. 8), MATP-I cannot achieve the same performance as MATP-P even if $d_{\mathrm{SU}_{0}}=40 \mathrm{~km}$. On the other hand, MATP-C can achieve the same performance as MATP-P in the region where $d_{\mathrm{SU}_{0}}>20 \mathrm{~km}$. These observations also relate the result shown in Fig. 4. In the case of MATP-I, $\operatorname{Pr}\left(\mathrm{D}_{T}=1\right)$ is still less than 0.5 at $d_{\mathrm{SU}_{0}}=40 \mathrm{~km}$. This result implies that the MATP-C outperforms the MATP-I for larger $\sigma_{x}$.

The results also show that the rate of increase of $\bar{C}_{\text {down }}$ depends on $\sigma_{x}$, where a smaller $\sigma_{x}$ leads to a larger rate of

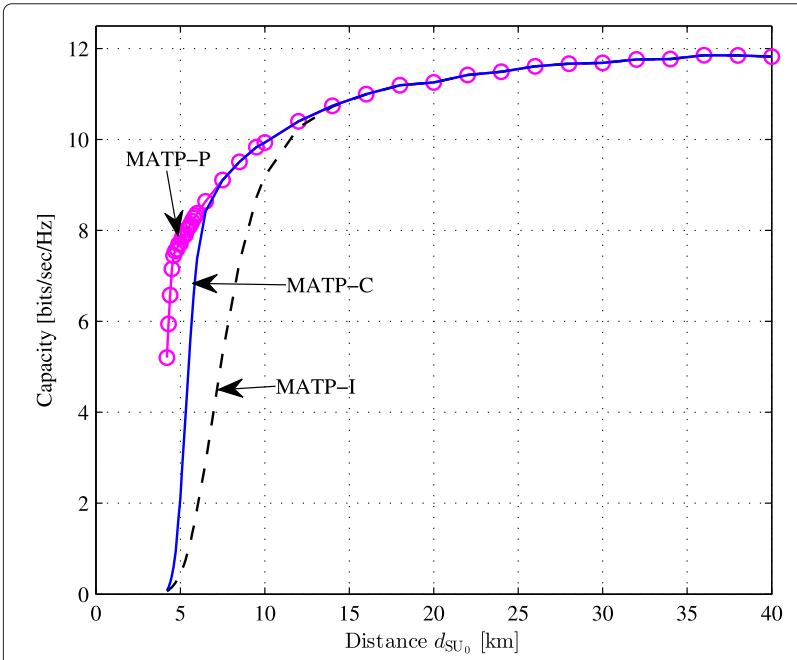

Fig. 7 The capacity $\bar{C}_{\text {down }}$ as a function of $d_{S U_{0}}$ for MATP-P, MATP-C $\left(N_{S}=4\right)$, and MATP-I, with $\dot{P}_{C}=0.01, N_{S}=4, \eta=3, \sigma_{x}=3 \mathrm{~dB}$, $r_{C, P}=3.68 \mathrm{~km}, r_{C, S}=500 \mathrm{~m}$, and $d_{g}=4.2 \mathrm{~km}$

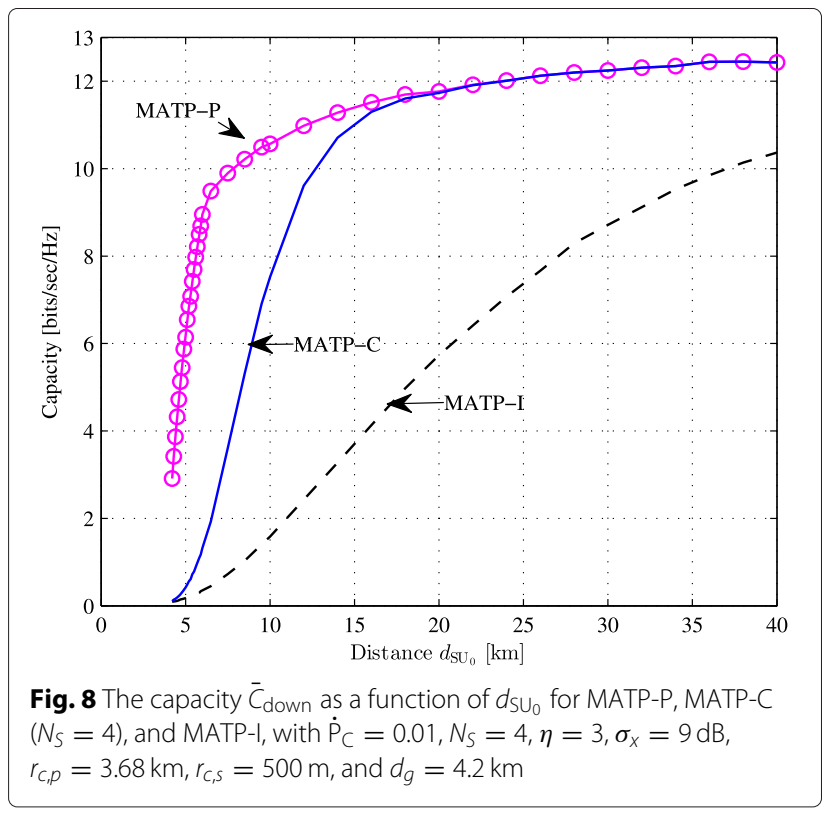

increase. We can conclude based on the results that cooperative measurement is an effective approach especially when $\sigma_{x}$ is large.

\subsection{Impact of $N_{s}$ in the cooperative approach}

In order to evaluate the effects of $N_{s}$ on the cooperative measurement, we evaluate MATP-C and MATP-I in terms of $\bar{C}_{\text {down }}$ as a function of $N_{s}$. The results are shown in Fig. 9. In this evaluation, $\sigma_{x}=9 \mathrm{~dB}$ and $d_{g}=4.2 \mathrm{~km}$.

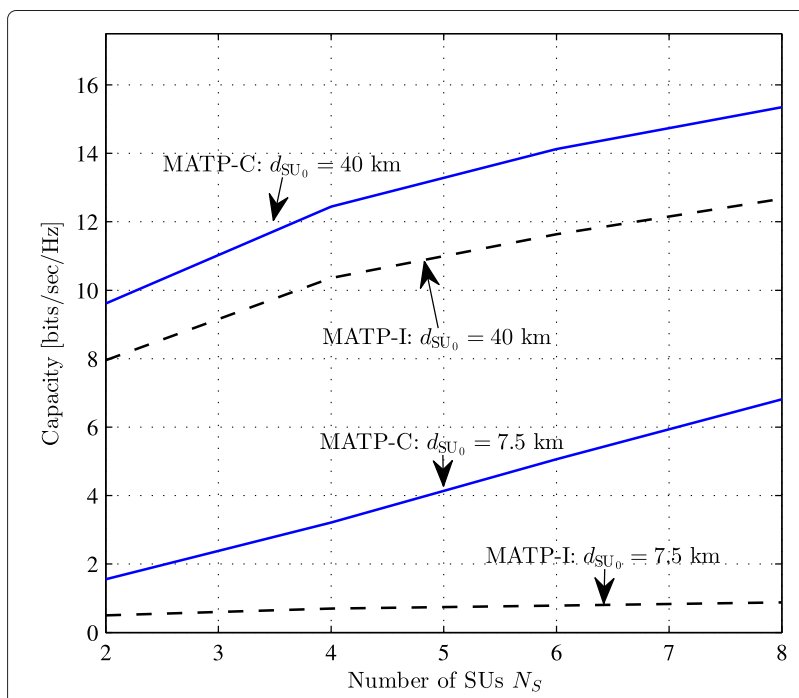

Fig. 9 The capacity $\bar{C}_{\text {down }}$ as a function of the number of SUs $N_{s}$ for MATP-C and MATP-I and for $d_{S U_{0}}=7.5$ and $d_{S U_{0}}=40 \mathrm{~km}$ with $\dot{\mathrm{P}}_{\mathrm{C}}=0.01, \eta=3, \sigma_{X}=9 \mathrm{~dB}, r_{c, p}=3.68 \mathrm{~km}, r_{C, S}=500 \mathrm{~m}$, and $d_{g}=4.2 \mathrm{~km}$ 
In the case of MATP-I at $d_{\mathrm{SU}_{0}}=7.5 \mathrm{~km}$, increasing $N_{S}$ does not have a significant impact. In comparison, the MATP-C significantly benefits from increasing $N_{S}$.

The MATP-C benefits from the cooperative measurement and similar to the MATP-I from the multiuser diversity gain in the power allocation process [42]. In the case of $d_{\mathrm{SU}_{0}}=40 \mathrm{~km}$, the rate of increase of MATP-C is slightly larger than that of MATP-I. This gain seen in MATP-C and MATP-I is mainly attributed to the multiuser diversity gain.

\subsection{Impact of extended PN coverage $d_{g}$}

In the discussion of the results of Figs. 5 and 6, we stated that setting $d_{g}$ is an important issue in both MATP-I and MATP-C, since there is a trade-off between the additional separation radius $\Delta d_{g}$ and the transmission power margin $T_{\mathrm{m}}^{(Z) *}$. Specifically, a larger $d_{g}$ is equivalent to a larger $\Delta d_{g}$, which leads to a reduced area where the spectrum can be used by the SB. A smaller $d_{g}$ leads to a larger $T_{\mathrm{m}}^{(Z) *}$, as shown by Fig. 6 .

To confirm this effect in $\bar{C}_{\text {down }}$, we plot $\bar{C}_{\text {down }}$ as a function of $d_{\mathrm{SU}_{0}}$ in terms of different $d_{g}\left(d_{g}=3.7, d_{g}=\right.$ 4.2, and $\left.d_{g}=5 \mathrm{~km}\right)$ in Fig. 10. This result demonstrates that the $\bar{C}_{\text {down }}$ also depends on $d_{g}$. In both MATP-C and MATP-I, the case when $d_{g}=4.2 \mathrm{~km}$ achieves the best $\bar{C}_{\text {down }}$ performance.

The curve when $d_{g}=5 \mathrm{~km}$ is almost an exact duplicate of the curve for $d_{g}=4.2 \mathrm{~km}$, but shifted to the right. This is because the difference in $\Delta d_{g}$ is dominant, which causes the gap between the $\bar{C}_{\text {down }}$ performance of $d_{g}=5 \mathrm{~km}$ and that of $d_{g}=4.2 \mathrm{~km}$. On the other hand, we can confirm that there is a significant performance gap between $d_{g}=3.7$ and $d_{g}=4.2 \mathrm{~km}$, which is mainly attributed to the difference in the margin, $T_{\mathrm{m}}^{*}$.

\subsection{Optimum point in terms of extended PN coverage $d_{g}$}

The average capacity performances depend on the extended PN coverage, $d_{g}$, as confirmed by the result of Fig. 10. We define $\bar{C}_{\text {down }}$ as a function of distance, $d_{\mathrm{SU}_{0}}$, as $\bar{C}_{\mathrm{down}}\left(d_{\mathrm{SU}_{0}}\right)$, and define a new metric to evaluate the MATPs as

$\bar{C}_{\text {down }}^{*}=\frac{1}{\left|d_{\max }-r_{c, p}\right|} \int_{d_{\mathrm{SU}_{0}}=r_{c, p}}^{d_{\mathrm{SU}_{0}}=d_{\mathrm{max}}} \bar{C}_{\mathrm{down}}\left(d_{\mathrm{SU}_{0}}\right) d d_{\mathrm{SU}_{0}}$,

where $d_{\max }$ indicates an assumed maximum distance. This corresponds to $d_{\mathrm{SU}_{0}}$ having uniform distribution in the region $r_{c, p} \leq d_{\mathrm{SU}_{0}} \leq d_{\max }$. In the evaluation performed here, we use $d_{\max }=40 \mathrm{~km}$. This metric $\bar{C}_{\text {down }}^{*}$ indicates the average of $\bar{C}_{\text {down }}\left(d_{\mathrm{SU}_{0}}\right)$ in the $d_{\mathrm{SU}_{0}}$ domain.

Figure 11 shows $\bar{C}_{\text {down }}^{*}$ as a function of $d_{g}$ for MATP-C and MATP-I. In both cases, proper $\bar{C}_{\text {down }}^{*}$ can be achieved around $d_{g}=4.2 \mathrm{~km}$. The smallest $d_{g}$ is $r_{c, p}=3.68 \mathrm{~km}$, and it may lead to much space in which operation of SN is available. However, the result in Fig. 11 indicates that small $d_{g}$ is not in fact favorable from the perspective of network capacity. From the perspective of the protection of $\mathrm{PN}$, longer $d_{g}$ is obviously favorable.

\section{Conclusions}

In this paper, we investigated methods to set the MATP for SS. In our proposed approach, the SB sets MATP based on an estimate of the distance between the $\mathrm{SB}$ and $\mathrm{PB}$ (transmitter). We compared against MATP-P where the location information is available at the SB.

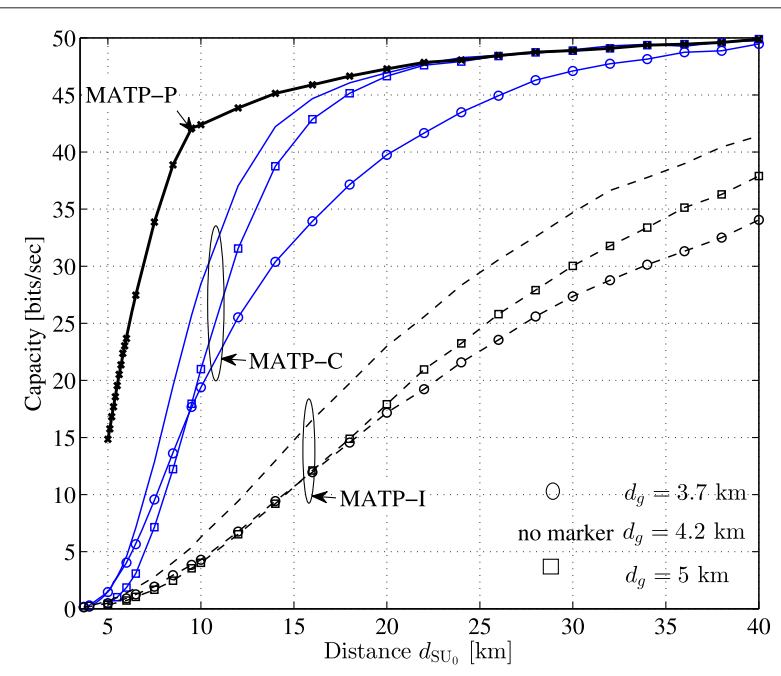

Fig. 10 The capacity $\bar{C}_{\text {down }}$ as a function of distance $d_{S U_{0}}$ for $d_{g}=3.7, d_{g}=4.2$, and $d_{g}=5 \mathrm{~km}$. MATP-P and MATP-C $\left(N_{S}=4\right)$, with $\dot{P}_{C}=0.01, \eta=3, \sigma_{x}=9 \mathrm{~dB}, r_{c, p}=3.68 \mathrm{~km}$, and $r_{c, s}=500 \mathrm{~m}$

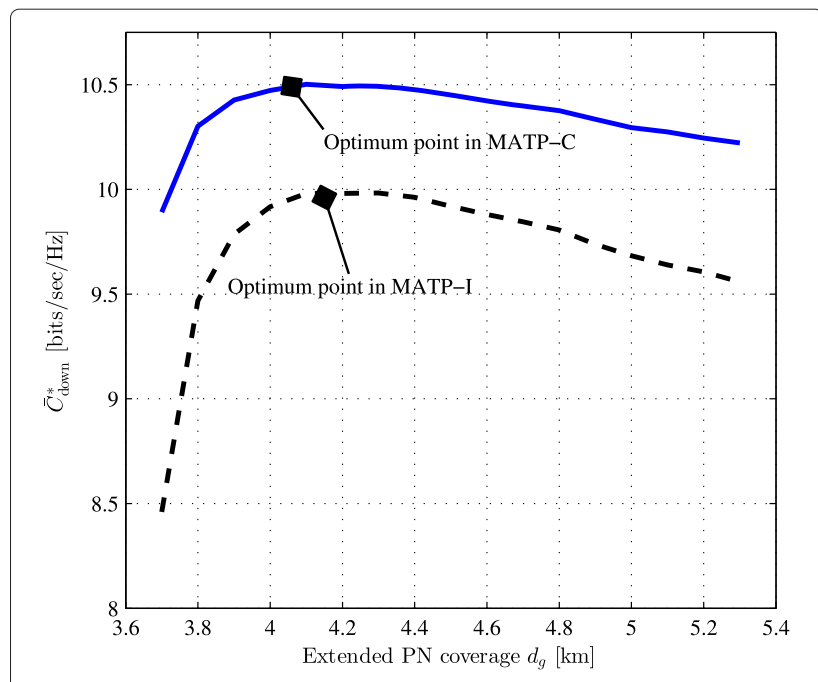

Fig. $11 \bar{C}_{\text {down }}^{*}$ as a function of extended PN coverage $d_{g}$ for MATP-C $\left(N_{S}=4\right)$ and MATP-l, with $\dot{P}_{C}=0.01, \eta=3, \sigma_{x}=9 \mathrm{~dB}, r_{c, p}=3.68 \mathrm{~km}$, $r_{c, S}=500 \mathrm{~m}$, and $d_{\max }=40 \mathrm{~km}$ 
To satisfy the CTP, the SB has to consider three issues: shadowing from the PB (transmitter) to the SU, shadowing from the SB to the PU receiver, and the lack of location information. To handle these issues, we proposed a two-step approach to set the MATP where two margins, the transmission decision margin and the transmit power margin, were employed. The former is to guarantee the protection of the PUs when the SB resides within the extended PU network (PN) coverage, and the latter is to guarantee the PU protection when the SB resides outside of the extended PN coverage. We set the margins based on the analysis.

Numerical results verified our approach and showed that MATP-I and the MATP-C can satisfy the constraint for any placement of the SN. Furthermore, in MATP-C, cooperative measurements are used and the numerical results demonstrated that MATP-C always outperforms MATP-I in terms of average SN capacity. Setting the extended $\mathrm{PN}$ coverage range is also important since it significantly affects the average capacity, as was shown by the numerical results. In addition, we demonstrated that there is also an optimum range for the extended PN coverage that can maximize average capacity performance.

\section{Acknowledgements}

The work of Kenta Umebayashi was supported by JSPS KAKENHI Grant Number 15K06053. The work of J. Lehtomäki was supported by the SeCoFu project of the Academy of Finland.

\section{Competing interests}

The authors declare that they have no competing interests.

\section{Author details \\ ${ }^{1}$ Department of Electrical and Electronic Engineering, Tokyo University of Agriculture and Technology, Tokyo, Japan. ${ }^{2}$ Centre for Wireless Communications (CWC), University of Oulu, Oulu 90014, Finland. ${ }^{3}$ Department} of Communications Engineering (DCE), University of Oulu, Oulu 90014, Finland.

Received: 14 January 2016 Accepted: 14 May 2016

Published online: 06 June 2016

\section{References}

1. Spectrum policy task force report (2002). Technical Report Docket No. 02-135, FCC (Federal Communications Commission)

2. J Mitola, GQ Maguire, Cognitive radio: making software radios more personal. IEEE Personal Commun. Mag. 6, 13-18 (1999)

3. S Haykin, Cognitive radio: brain-empowered wireless communications. IEEE J. Sel. Areas Commun. 23(2), 201-220 (2005)

4. IF Akyildiz, W-Y Lee, MC Vuran, S Mohanty, Next generation/dynamic spectrum access/cognitive radio wireless networks: a survey. Comput. Netw. Int. J. Comput. Telecommun. Netw. 50, 2127-2159 (2006)

5. R Zhang, YC Liang, S Cui, Dynamic resource allocation in cognitive radio networks. IEEE Signal Process. Mag. 27(3), 102-114 (2010)

6. R Zhang, On active learning and supervised transmission of spectrum sharing based cognitive radios by exploiting hidden primary radio feedback. IEEE Trans. Commun. 58(10), 2960-2970 (2010)

7. N Hoven, A Sahai, in Proc. Wireless Networks, Communications and Mobile Computing. Power scaling for cognitive radio, (HI, USA, 2005), pp. 250-255

8. K Hamdi, Z Wei, KB Letaief, in Proc. IEEE International Conference on Communications (ICC). Power control in cognitive radio systems based on spectrum sensing side information, (Glasgow, Scotland, 2007), pp. 24-28

9. W Xin, Joint sensing-channel selection and power control for cognitive radios. IEEE Trans. Wirel. Commun. 10(3), 958-967 (2011)
10. V Asghari, S Aissa, Resource management in spectrum-sharing cognitive radio broadcast channels: adaptive time and power allocation. IEEE Trans. Commun. 59(5), 1446-1457 (2011)

11. T Yucek, H Arslan, A survey of spectrum sensing algorithms for cognitive radio applications. IEEE Commun. Surv. Tutor. 11, 116-130 (2009)

12. SM Mishra, A Sahai, RW Brodersen, in Proc. IEEE International Conference on Communications (ICC). Cooperative sensing among cognitive radios, ( Turkey, 2006), pp. 1658-1663

13. S Srinivasa, SA Jafar, Soft sensing and optimal power control for cognitive radio. IEEE Trans. Wireless Commun. 9(12), 3638-3649 (2010)

14. R Zhang, YC Liang, Exploiting multi-antennas for opportunistic spectrum sharing in cognitive radio networks. IEEE J. Sel. Areas Commun. 2(1), 88-102 (2008)

15. L Musavian, S Aissa, Capacity and power allocation for spectrum sharing communications in fading channels. IEEE Trans. Wirel. Commun. 8(1), 148-156 (2009)

16. R Zhang, S Cui, YC Liang, On ergodic sum capacity of fading cognitive multiple-access and broadcast channels. IEEE Trans. Inf. Theory. 55(11), 5161-5178 (2009)

17. FF Digham, in Proc. IEEE Wireless Communications and Networking Conference (WCNC). Joint power and channel allocation for cognitive radios, ( Nevada, USA, 2008), pp. 882-887

18. SY Lee, SH An, YM Yoon, in Proc. International Conference on Advanced Communication Technology (ICACT). Area spectrum efficiency of TV white space wireless system with transmit power control, (Gangwon-Do, Korea, 2010), pp. 1061-1066

19. E Dall'Anese, S-J Kim, GB Giannakis, S Pupolin, Power control for cognitive radio networks under channel uncertainty. IEEE Trans. Wirel. Commun. 10(10), 3541-3551 (2011)

20. ECY Peh, Y-C Liang, Y Zeng, in Communication Systems (ICCS), 2012 IEEE International Conference On. Sensing and power control in cognitive radio with location information, (Nebraska, USA, 2012), pp. 255-259

21. S Sorooshyari, CW Tan, M Chiang, Power control for cognitive radio networks: axioms, algorithms, and analysis. IEEE/ACM Trans. Networking. 20(3), 878-891 (2012)

22. SM Sanchez, RD Souza, EMG Fernandez, VA Reguera, Rate and energy efficient power control in a cognitive radio ad hoc network. IEEE Signal Proc. Lett. 20(5), 451-454 (2013)

23. S Gong, P Wang, Y Liu, W Zhuang, Robust power control with distribution uncertainty in cognitive radio networks. IEEE J. Sel. Areas Commun. 31(11), 2397-2408 (2013)

24. J Wang, J Chen, Y Lu, M Gerla, D Cabric, Robust power control under location and channel uncertainty in cognitive radio networks. IEEE Wirel. Commun. Lett. 4(2), 113-116 (2015)

25. HA Suraweera, PJ Smith, M Shafi, Capacity limits and performance analysis of cognitive radio with imperfect channel knowledge. IEEE Trans. Veh. Technol. 59(4), 2960-2970 (2010)

26. S Huang, X Liu, Z Ding, in Proc. IEEE International Conference on Computer Communications (INFOCOM). Distributed power control for cognitive user access based on primary link control feedback, (CA, USA, 2010), pp. $1280-1288$

27. K Muraoka, H Sugahara, M Ariyoshi, in Proc. IEEE Dynamic Spectrum Access Networks International Symposium (DySPAN). Monitoring-based spectrum management for expanding opportunities of white space utilization, (Aachen, Germany, 2011), pp. 277-284

28. H Nam, MB Ghorbel, M-S Alouini, in Proc. Cognitive Radio Oriented Wireless Networks \& Communications (CROWNCOM). Location-based resource allocation for OFDMA cognitive radio systems, (Cannes, France, 2010), pp. 1-5

29. MB Ghorbel, H Nam, M-S Alouini, in Proc. Wireless Communication Systems (ISWCS). Generalized location-based resource allocation for OFDMA cognitive radio systems, (2010), pp. 1011-1016

30. V Asghari, S Aïssa, in Proc. IEEE Global Telecommunications Conference (GLOBECOM). Resource sharing in cognitive radio systems: outage capacity and power allocation under soft sensing, (LA, USA, 2008), pp. 1-5

31. M Hong, J Kim, H Kim, Y Shin, in Proc. IEEE Consumer Communications and Networking Conference (CCNC). An adaptive transmission scheme for cognitive radio systems based on interference temperature model, (Nevada, USA, 2008), pp. 69-73

32. S Kim, H Jeon, J Ma, in Proc. IEEE Military Communications Conference (MILCOM). Robust localization with unknown transmission power for cognitive radio, (FL, USA, 2007), pp. 1-6 
33. Z Ma, W Chen, KB Letaief, Z Cao, A semi range-based iterative localization algorithm for cognitive radio networks. IEEE Trans. Veh. Technol. 59(2), 704-717 (2010)

34. N Patwari, JN Ash, S Kyperountas, AO Hero, RL Moses, NS Correal, Locating the nodes: cooperative localization in wireless sensor networks. IEEE Signal Process. Mag. 22(4), 54-69 (2005)

35. M Hideyuki, Y Ryoji, M Hideo, O Tomoyoshi, O Seizo, MMSE mobile station positioning method using signal strength in cellular systems. IEICE Trans. Fundam. Electron. Comput. Sci. 86(7), 1593-1602 (2003)

36. M Mishra, A Sahai, How much white space is there? Technical report UCB/EECS-2009-3, EECS Department, University of California, Berkeley (2009). http://www.eecs.berkeley.edu/Pubs/TechRpts/2009/ EECS-2009-3.html

37. $M V u, N$ Devroye, $V$ Tarokh, On the primary exclusive region of cognitive networks. Trans. Wireless. Comm. 8(7), 3380-3385 (2009)

38. TS Rappaport (ed.), Wireless Communications: Principles \& Practice. Prentice Hall (Prentice Hall, Englewood Cliffs, 2002)

39. SM Kay, Fundamentals of Statistical Signal Processing, Volume 2: Detection Theory. (Prentice Hall PTR, New York, 1998)

40. J Zheng, Cooperative localization based on received signal strength in wireless sensor network. Master's thesis, University of Ontario Institute of Technology (2010)

41. SD Chitte, S Dasgupta, Z Ding, Distance estimation from received signal strength under log-normal shadowing: bias and variance. IEEE Signal Process. Lett. 16(3), 216-218 (2009)

42. D Tse, P Viswanath (eds.), Fundamentals of Wireless Communication (Cambridge University Press, Cambridge, 2005)

\section{Submit your manuscript to a SpringerOpen ${ }^{\circ}$ journal and benefit from:}

- Convenient online submission

- Rigorous peer review

- Immediate publication on acceptance

- Open access: articles freely available online

- High visibility within the field

- Retaining the copyright to your article

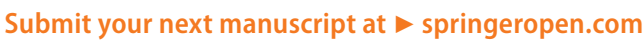

